\title{
Treatment of Orthostatic Hypotension Due to Autonomic Dysfunction (Neurogenic Orthostatic Hypotension) in a Patient with Cardiovascular Disease and Parkinson's Disease
}

Peter A. McCullough

Received: September 13, 2018 / Published online: January 9, 2019

(C) The Author(s) 2019

\section{ABSTRACT}

Introduction: The prevalence of neurogenic orthostatic hypotension $(\mathrm{nOH})$ increases with age and is associated with autonomic failure in neurodegenerative diseases (e.g., Parkinson's disease). Symptoms can interfere with daily activities that require standing or walking and can increase risk of falls and related morbidity. Many patients with nOH have or develop cardiovascular comorbidities that can predate $\mathrm{nOH}$ symptoms or may arise as a result of autonomic dysregulation. In this report, we describe a complicated case of a patient with cardiovascular disease and Parkinson's disease who presented with orthostatic symptoms.

Case Report: A 78-year-old man with a history of coronary heart disease, class III heart failure, cardiac cachexia, long-standing persistent atrial fibrillation (AF), Hodgkin's lymphoma, and Parkinson's disease presented with weakness, dizziness, presyncope, fatigue, and inability to stand. Orthostatic blood pressure (BP) measurements revealed a seated $\mathrm{BP}$ of $120 / 70 \mathrm{mmHg}$ that decreased to $60 / 40 \mathrm{mmHg}$ upon standing, accompanied by a slight

Enhanced digital features To view enhanced digital features for this article go to https://doi.org/10.6084/ m9.figshare.7498049.

P. A. McCullough $(\bowtie)$

Baylor University Medical Center, Dallas, TX, USA e-mail: peteramccullough@gmail.com increase in heart rate from 70 to 74 beats per minute. He was diagnosed with $\mathrm{nOH}$ and prescribed droxidopa (titrated to $600 \mathrm{mg}$ three times daily). Treatment with droxidopa improved the patient's ability to stand and his orthostatic BP.

Conclusion: Droxidopa is approved by the US Food and Drug Administration to treat symptomatic $\mathrm{nOH}$ and is not contraindicated in patients with cardiovascular conditions. In this case, treatment with droxidopa improved the patient's orthostatic tolerance and, importantly, did not change the patient's rate-controlled AF or his symptoms of class IV heart failure. Because symptoms associated with $\mathrm{nOH}$ can be detrimental to patient safety and mobility, it is critical to screen for and treat patients with $\mathrm{nOH}$, even when there are cardiovascular comorbidities.

Funding: Editorial support and article processing charges were funded by Lundbeck.

Plain Language Summary: Plain language summary available for this article.

Keywords: Blood pressure; Cardiovascular disease; Diagnosis; Heart failure; Neurogenic orthostatic hypotension; Screening; Treatment

\section{PLAIN LANGUAGE SUMMARY}

A 78-year-old man who had heart disease and Parkinson's disease visited his cardiologist 
because he felt weak, dizzy, lightheaded, and tired and was unable to stand up. His doctor took his blood pressure when he was seated and again when he stood up. These measurements showed that his blood pressure was normal while seated but greatly decreased upon standing. Based on his symptoms and blood pressure results, the man was diagnosed with neurogenic orthostatic hypotension ( $\mathrm{nOH}) . \mathrm{nOH}$ is a medical condition that occurs when blood pressure decreases too much when a person changes from a seated or lying position to an upright position. People who are older or have medical conditions related to nervous system failure, such as Parkinson's disease, are at greater risk of having $\mathrm{nOH}$. This patient was given the drug droxidopa to treat his nOH symptoms. Once he was treated, his symptoms improved, and he was better able to tolerate standing. The most common symptom of $\mathrm{nOH}$ is dizziness or lightheadedness when standing. People who feel this way may have a hard time standing and walking and are more likely to fall, which could cause a serious injury. It is important for people with symptoms such as weakness, dizziness, or lightheadedness when standing to discuss them with their healthcare provider. Their healthcare provider can then screen for $\mathrm{nOH}$ and provide treatment options if necessary.

\section{INTRODUCTION}

Neurogenic orthostatic hypotension $(\mathrm{nOH})$ is defined as a sustained decrease in blood pressure (BP) within 3 min of standing or head-up tilt to $60^{\circ}$ on a tilt table (decrease of $\geq 20 \mathrm{mmHg}$ systolic or $\geq 10 \mathrm{mmHg}$ diastolic) [1]. The prevalence of $\mathrm{nOH}$ increases with age and in patients who have disorders associated with autonomic failure (e.g., Parkinson's disease, multiple system atrophy, pure autonomic failure, and dementia with Lewy bodies) [2]. Symptoms of nOH include dizziness or lightheadedness, syncope or presyncope, problems with vision, generalized weakness, fatigue, trouble concentrating, and head or neck discomfort [3]. These symptoms can interfere with activities of daily living that require standing or walking and can also increase risk of falls and fall-related morbidity [3, 4].
A complicating factor for the treatment of patients with $\mathrm{nOH}$ is that many of them have or will develop cardiovascular comorbidities, including hypertension, atrial fibrillation (AF), and heart failure [5]. These comorbidities can predate $\mathrm{nOH}$ symptoms or may arise as a result of autonomic dysregulation and upregulation of other mechanisms that control arterial tone to maintain circulatory homeostasis, such as the renin-angiotensin system, endothelin, and arginine vasopressin [6-9]. The renin-angiotensin system, in particular, may influence supine hypertension $[7,8]$. Approximately half of patients with autonomic failure may also have supine hypertension (BP $\geq 140 \mathrm{mmHg}$ systolic or $\geq 90 \mathrm{mmHg}$ diastolic), complicating treatment of these hemodynamic opposites $[6,10,11]$. In this report, we describe a patient with cardiovascular disease and Parkinson's disease who presented with orthostatic symptoms, which complicated his treatment course.

\section{CASE PRESENTATION}

A 78-year-old man with a 2-year history of class III heart failure and a 10-year history of Parkinson's disease presented to his cardiologist's office with weakness, dizziness, presyncope, fatigue, and inability to stand. He had experienced cardiac cachexia within the past 2 years, with his weight decreasing from $146 \mathrm{lbs}$ to as low as 109 lbs. Additionally, his cardiac history included a left ventricular ejection fraction of 35\%, long-standing persistent $\mathrm{AF}$, anemia, and coronary heart disease. The patient also had a history of Hodgkin lymphoma and chronic lymphocytic leukemia, which had been treated 8 years earlier using chemotherapy and radiation; the radiation exposure resulted in restrictive lung disease.

The patient's medications included carvedilol (6.25 mg twice daily [BID]), enalapril $(2.5 \mathrm{mg}$ BID), digoxin (0.125 mgpo QD), furosemide (20 $\mathrm{mg}$, as needed), and warfarin (2.5 $\mathrm{mg}$ once daily [QD]) for cardiac symptoms, as well as levodopa/ carbidopa (25/100 mg four times daily) for Parkinson's disease symptoms and sertraline (50 mg QD) for mood regulation. He was also taking tolvaptan (15 mg QD) to treat recurrent symptomatic hyponatremia. 
Physical examination revealed a seated BP of $120 / 70 \mathrm{mmHg}$ that decreased to $60 / 40 \mathrm{mmHg}$ upon standing, meeting the diagnostic criteria for orthostatic hypotension. His heart rate increased only slightly, from 70 beats per minute (bpm) seated to $74 \mathrm{bpm}$ upon standing; this increase $(<15 \mathrm{bpm})$ is consistent with a diagnosis of $\mathrm{nOH}$. A full review of his medications indicated that several medications, including carvedilol, enalapril, and furosemide, could be contributing to his orthostatic symptoms; however, they were at the minimum effective doses and were indicated for treatment of heart failure with reduced ejection fraction. Consequently, no washout period of the patient's cardiac medications was conducted to assess for any effects on orthostatic hypotension symptoms. His serum creatinine and blood urea nitrogen were normal, and there was no evidence of acute or chronic kidney disease throughout the course of his illness. The hemoglobin, total serum iron, iron saturation, and ferritin values revealed anemia but no iron deficiency. Evaluations of 24-h ambulatory BP and Holter monitor measurements were not performed. The combination of his heart failure, restrictive lung disease, and overall cachexia were so severe that he was deemed not to be a candidate for an implantable cardio-defibrillator or advanced mechanical circulatory support. Over time, he developed symptoms of class IV heart failure.

The patient was prescribed $200 \mathrm{mg}$ droxidopa (three times daily [TID]) to treat his orthostatic symptoms. The dose was increased to $600 \mathrm{mg}$ TID for better control of $\mathrm{nOH}$ symptoms. Droxidopa, a norepinephrine prodrug, is approved by the US Food and Drug Administration to treat symptomatic $\mathrm{nOH}$ in adults [12]. The patient reported feeling better and less likely to fall when standing. During an office visit, his BP changed from 124/82 mmHg supine to $110 / 76 \mathrm{mmHg}$ upon standing with assistance. There were no changes in his ratecontrolled AF or symptoms of class IV heart failure, and his weight remained low but stable. The patient was deceased at the time of case study preparation, and informed consent was obtained from his next of kin.

\section{DISCUSSION}

The effect of nOH symptoms on patient mobility and safety necessitates efficient diagnosis and treatment [3]. Patients with symptomatic $\mathrm{nOH}$ are at an increased risk of syncope and falls, with consequences such as injury [13], hospitalization, increased healthcare costs [14], increased likelihood of admission to a skilled nursing facility [15], and even death [16]. The patient described in this case study had longstanding persistent AF (AF lasting longer than 12 months), symptoms of which overlap with those characteristic of $\mathrm{nOH}$, including dizziness, fatigue, and dyspnea [1, 17]. Additionally, AF treatment with antiarrhythmic agents such as amiodarone, verapamil, propafenone, sotalol, or flecainide can result in peripheral polyneuropathy, which may exacerbate symptoms of nOH such as weakness $[18,19]$. In the current case study, the patient was very frail, and treatment of $\mathrm{nOH}$ was important to reduce the risk of falls and related morbidity. Droxidopa therapy was effective for treatment of the symptoms of $\mathrm{nOH}$ (no observations were made regarding the effects on symptoms of Parkinson's disease) and did not adversely affect the patient's rate-controlled $\mathrm{AF}$, nor were any adjustments to concomitant medications for the management of cardiovascular comorbidities required.

Patients with $\mathrm{nOH}$ also often have cardiovascular comorbidities, and droxidopa can be used to treat symptomatic $\mathrm{nOH}$ without compromising cardiovascular safety. Droxidopa is not contraindicated in patients taking medications to control AF, as in the present case, or other cardiovascular conditions, although it may exacerbate some existing cardiovascular conditions [12]. An analysis of the cardiovascular safety of droxidopa in patients with symptomatic $\mathrm{nOH}$ demonstrated comparable rates of cardiovascular events between patients receiving droxidopa and patients receiving placebo [20]. In a clinical trial with up to 10 weeks' exposure to droxidopa, 70\% of patients had preexisting cardiovascular disease; among this patient subgroup, cardiovascular adverse event rates were low in the droxidopa cohort $(6.3 \%[n=5 / 79])$ but slightly higher than 
in the placebo cohort $(2.6 \%[n=2 / 77])$ [20]. Furthermore, droxidopa did not affect cardiac depolarization (QTc interval), heart rate, atrioventricular conduction (PR interval), or ventricular depolarization (QRS interval) in healthy adults [21].

Diabetes mellitus is a common comorbidity in patients with cardiovascular disease. Although there are some similarities between the neuropathy observed in diabetes mellitus and disorders associated with primary autonomic failure [22], there are limited data on the use of droxidopa in patients with diabetic autonomic neuropathy, because patients with nOH due to diabetic autonomic neuropathy were excluded from the clinical studies leading to US Food and Drug Administration approval. These clinical trials included a few patients with primary autonomic failure and coexistent diabetes mellitus, but no analyses of the diabetic subpopulation were performed. Examination of the effect of droxidopa in patients with $\mathrm{nOH}$ due to diabetic autonomic neuropathy is a potential area for future study.

Medications used to treat $\mathrm{nOH}$ necessarily increase BP, which can increase the risk of supine hypertension [6]. Supine hypertension may be managed using measures like elevating the head of the bed for sleep and not resting in the supine position during the day [3]. Shortacting antihypertensives may be needed at bedtime to treat severe supine hypertension, although their use increases the risk of $\mathrm{nOH}$ symptoms during nighttime trips to the bathroom [3]. Medications used to treat hypertension and other cardiac conditions, including diuretics, beta-adrenergic blockers, and angiotensin-converting enzyme inhibitors, can worsen symptoms of $\mathrm{nOH}$ and increase the shortterm risk of syncope and falls; the use of these medications must, therefore, be monitored carefully and adjusted as necessary $[3,6]$. Because patients with $\mathrm{nOH}$ may be older, have multiple comorbid conditions, and use polypharmacy, they may be medically complex and receive care from multiple healthcare providers in various specialties (e.g., cardiology, neurology, primary care). The management of $\mathrm{nOH}$ symptoms is important in order to reduce symptomatic burden and improve the patient's ability to perform daily activities that involve standing. Patient safety is a key consideration, especially when treating persons who may have an increased risk for falls and injuries (e.g., those who are older or frail or who have Parkinson's disease), such as the individual in this case. However, treatment should be individualized to each patient based on his or her circumstances and coexisting conditions, and this may require collaboration among the care teams. Optimal management of $\mathrm{nOH}$ may require an integrated team approach among all healthcare providers caring for the patient, so that the benefits (e.g., improvement of $\mathrm{nOH}$ symptoms) and risks (e.g., exacerbation of any comorbidities) can be balanced appropriately.

\section{CONCLUSION}

Despite the complexity of treating $\mathrm{nOH}$ in patients with cardiovascular comorbidities, it is critical to screen for and treat $\mathrm{nOH}$ in patients with autonomic dysfunction and orthostatic symptoms. The balancing of the treatment of symptomatic nOH while appropriately and effectively managing concomitant cardiovascular disease is a clinical challenge requiring knowledge of the $\mathrm{nOH}$ disease state.

\section{ACKNOWLEDGEMENTS}

Funding. Lundbeck provided funding for editorial support for manuscript preparation and article processing charges but did not fund any other aspect of the case study. The author had full access to all of the data in this study and takes complete responsibility for the integrity of the data and accuracy of the data analysis.

Authorship. The author meets the International Committee of Medical Journal Editors (IMCJE) criteria for authorship for this article, takes responsibility for the integrity of the work as a whole, and has given his approval for this version to be published. 


\section{Medical Writing, Editorial, and Other} Assistance. Medical writing and editorial assistance in the preparation of this article was provided by Lauren Stutzbach, PhD, and Patrick Little, PhD, of the CHC Group (North Wales, PA, USA). Support for this assistance was funded by Lundbeck.

Disclosures. Peter A. McCullough has, in the past, received consulting fees from Lundbeck. Lundbeck had no role in conducting the case study or in the collection, analysis, or interpretation of data. The decision to submit this article for publication was made solely by the author.

Compliance with Ethics Guidelines. The patient was deceased at the time of manuscript preparation. Therefore, informed consent was obtained from his next of kin.

Data Availability. Data sharing is not applicable to this article, as no datasets were generated or analyzed during the current study.

Open Access. This article is distributed under the terms of the Creative Commons Attribution-NonCommercial 4.0 International License (http://creativecommons.org/licenses/ by-nc/4.0/), which permits any noncommercial use, distribution, and reproduction in any medium, provided you give appropriate credit to the original author(s) and the source, provide a link to the Creative Commons license, and indicate if changes were made.

\section{REFERENCES}

1. Freeman R, Wieling W, Axelrod FB, et al. Consensus statement on the definition of orthostatic hypotension, neurally mediated syncope and the postural tachycardia syndrome. Clin Auton Res. 2011;21(2):69-72.

2. Metzler M, Duerr S, Granata R, Krismer F, Robertson D, Wenning GK. Neurogenic orthostatic hypotension: pathophysiology, evaluation, and management. J Neurol. 2013;260(9):2212-9.

3. Gibbons $\mathrm{CH}$, Schmidt $\mathrm{P}$, Biaggioni $\mathrm{I}$, et al. The recommendations of a consensus panel for the screening, diagnosis, and treatment of neurogenic orthostatic hypotension and associated supine hypertension. J Neurol. 2017;264(8):1567-82.

4. Kaufmann H, Malamut R, Norcliffe-Kaufmann L, Rosa K, Freeman R. The Orthostatic Hypotension Questionnaire (OHQ): validation of a novel symptom assessment scale. Clin Auton Res. 2012;22(2):79-90.

5. Maule S, Milazzo V, Maule MM, Di Stefano C, Milan A, Veglio F. Mortality and prognosis in patients with neurogenic orthostatic hypotension. Funct Neurol. 2012;27(2):101-6.

6. Espay AJ, LeWitt PA, Hauser RA, Merola A, Masellis M, Lang AE. Neurogenic orthostatic hypotension and supine hypertension in Parkinson's disease and related synucleinopathies: prioritisation of treatment targets. Lancet Neurol. 2016;15(9):954-66.

7. Arnold AC, Okamoto LE, Gamboa A, et al. Mineralocorticoid receptor activation contributes to the supine hypertension of autonomic failure. Hypertension. 2016;67(2):424-9.

8. Baker J, Kimpinski K. Management of supine hypertension complicating neurogenic orthostatic hypotension. CNS Drugs. 2017;31(8):653-63.

9. Nilsson D, Sutton R, Tas W, Burri P, Melander O, Fedorowski A. Orthostatic changes in hemodynamics and cardiovascular biomarkers in dysautonomic patients. PLoS One. 2015;10(6):e0128962.

10. Shannon J, Jordan J, Costa F, Robertson RM, Biaggioni I. The hypertension of autonomic failure and its treatment. Hypertension. 1997;30(5):1062-7.

11. Umehara T, Matsuno H, Toyoda C, Oka H. Clinical characteristics of supine hypertension in de novo Parkinson disease. Clin Auton Res. 2016;26(1):15-21.

12. NORTHERA $^{\circledR}$ (droxidopa). Full Prescribing Information. Deerfield: Lundbeck NA Ltd; 2017.

13. Rudzinska M, Bukowczan S, Stozek J, et al. Causes and consequences of falls in Parkinson disease patients in a prospective study. Neurol Neurochir Pol. 2013;47(5):423-30.

14. Stevens JA, Corso PS, Finkelstein EA, Miller TR. The costs of fatal and non-fatal falls among older adults. Inj Prev. 2006;12(5):290-5.

15. Tinetti ME, Williams CS. Falls, injuries due to falls, and the risk of admission to a nursing home. $\mathrm{N}$ Engl J Med. 1997;337(18):1279-84.

16. Tinetti ME, Baker DI, McAvay G, et al. A multifactorial intervention to reduce the risk of falling 
among elderly people living in the community. N Engl J Med. 1994;331(13):821-7.

17. Nabauer M, Gerth A, Limbourg T, et al. The registry of the german competence network on atrial fibrillation: patient characteristics and initial management. Europace. 2009;11(4):423-34.

18. Antiarrhythmic drugs and polyneuropathy. The collaborative group for the study of polyneuropathy. J Neurol Neurosurg Psychiatry. 1994;57(3):340-3.

19. Wu C, Tcherny-Lessenot S, Dai W, et al. Assessing the risk for peripheral neuropathy in patients treated with dronedarone compared with that in other antiarrhythmics. Clin Ther. 2018;40(3):450-5.
20. White WB, Hauser RA, Rowse GJ, Ziemann A, Hewitt LA. Cardiovascular safety of droxidopa in patients with symptomatic neurogenic orthostatic hypotension. Am J Cardiol. 2017;119:1111-5.

21. White WB, Hewitt LA, Mehdirad AA. Impact of the norepinephrine prodrug droxidopa on the QTc interval in healthy individuals. Clin Pharmacol Drug Dev. 2018;7(3):332-40.

22. Freeman R, Abuzinadah AR, Gibbons C, Jones P, Miglis MG, Sinn DI. Orthostatic hypotension: JACC state-of-the-art review. J Am Coll Cardiol. 2018;72(11):1294-309. 